\title{
Disk-loss and disk-renewal phases in classical Be stars - II. Detailed analysis of spectropolarimetric data
}

\author{
Zachary H. Draper ${ }^{1}$, John P. Wisniewski ${ }^{1}$, Karen S. Bjorkman ${ }^{2}$, Jon \\ E. Bjorkman ${ }^{2}$, Xavier Haubois ${ }^{3}$, Alex C. Carciofi ${ }^{3}$, Marilyn R. \\ Meade $^{4}$ \\ ${ }^{1}$ Department of Astronomy, University of Washington, \\ email: zhd@u.washington.edu jwisnie@u.washington.edu \\ ${ }^{2}$ Ritter Observatory, Department of Physics \& Astronomy, University of Toledo \\ ${ }^{3}$ IAG, Universidade de Sao Paulo \\ ${ }^{4}$ Space Astronomy Lab, University of Wisconsin-Madison
}

\begin{abstract}
In Wisniewski et al. (2010), paper I, we analyzed 15 years of spectroscopic and spectropolarimetric data from the Ritter and Pine Bluff Observatories of 2 Be stars, 60 Cygni and $\pi$ Aquarii, when a transition from Be to B star occurred. Here we analyze the intrinsic polarization, where we observe loop-like structures caused by the rise and fall of the polarization Balmer Jump and continuum V-band polarization being mismatched temporaly with polarimetric outbursts. We also see polarization angle deviations from the mean, reported in paper I, which may be indicative of warps in the disk, blobs injected at an inclined orbit, or spiral density waves. We show our ongoing efforts to model time dependent behavior of the disk to constrain the phenomena, using 3D Monte Carlo radiative transfer codes.
\end{abstract}

Keywords. circumstellar matter, stars: individual ( $\pi$ Aquarii, 60 Cygni)

\section{Balmer Jump vs Continuum Polarization}

The time evolution of the intrinsic continuum V-band polarization (V-pol) of $\pi$ Aqr is shown in the left panel of Figure 1. We find evidence of clockwise loop-like structures (Fig. 1, middle panel) when comparing the evolution of the polarization across the Balmer Jump (BJ) vs V-pol, particularly during polarimetric outburst events (red in Fig. 1). $60 \mathrm{Cyg}$ also displays this behavior.
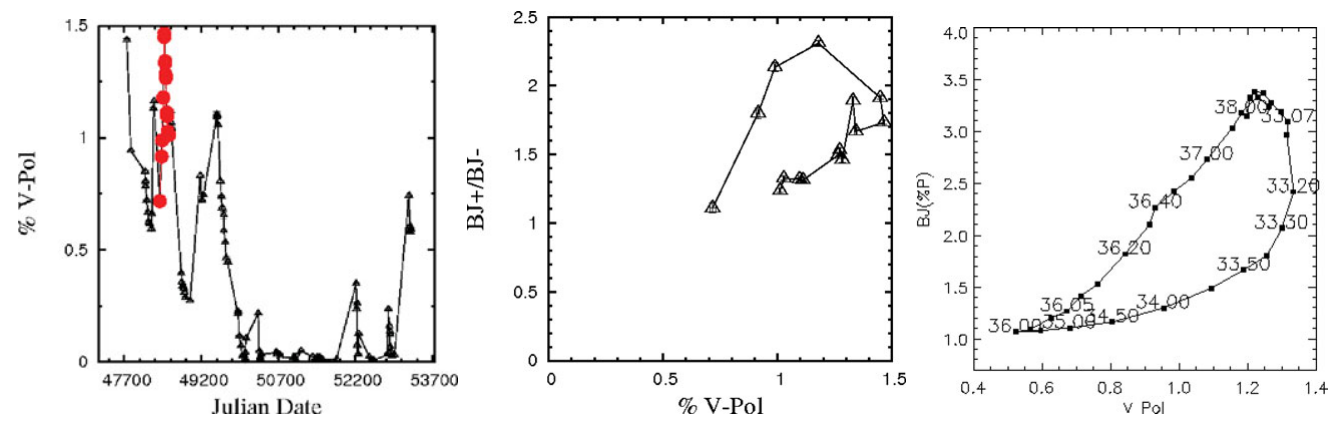

Figure 1. Left: Time evolution of the intrinsic continuum V-band polarization (V-pol) of $\pi$ Aqr; Middle: Loop-like structure of the evolution of the polarization across the Balmer Jump vs V-pol; Right: Modeled clockwise loop structures. 

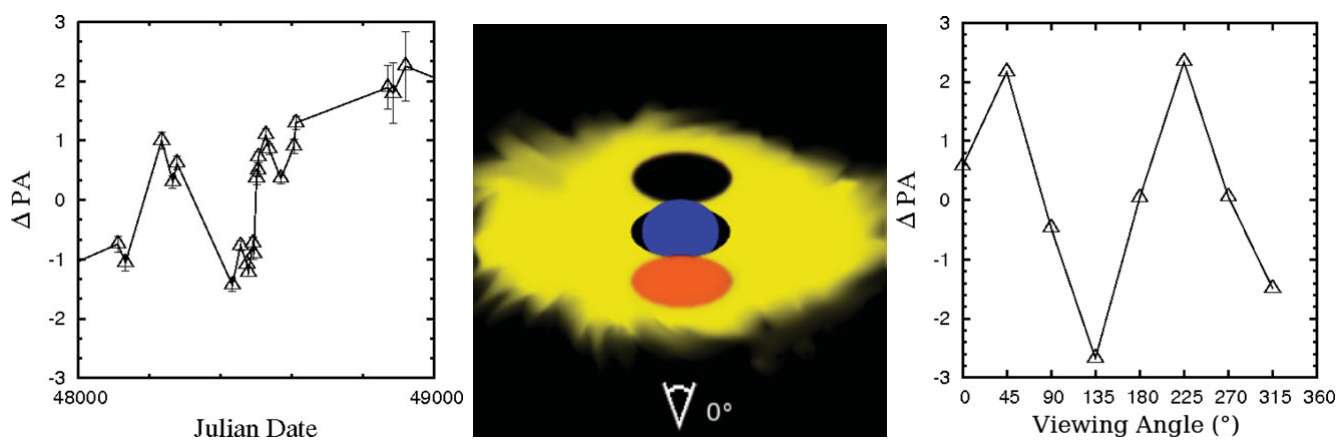

Figure 2. Left: Deviations in the mean PA during polarimetric outbursts of $\pi$ Aqr; Middle: Modelled density enhancement (orange) and decrement (black) on opposite sides of the disk; Right: Computed polarization from different viewing angles in a counter-clockwise motion.

\section{Modeling Polarization Loops}

We use the non-LTE 3D Monte Carlo code developed by Carciofi \& Bjorkman (2006), HDUST, to investigate the origin of the loop-like behavior of the BJ vs V-pol during polarimetric outbursts. Interestingly, we find that the clockwise loop structures can be reproduced when the mass-loss from the central star which feeds the disk is turned on (6 to 12 o clock, right panel of Fig. 1) then off (12 to 6 o' clock, right panel of Fig. 1). We therefore suggest that this diagnostic can provide insight into the time dependence of the density of the innermost disk region. Counter-clockwise loops are also observed in $\pi$ Aqr and $60 \mathrm{Cyg}$ and will require further modeling to ascertain thier origin. We note that de Wit et al. (2006) found similar loop structures in CMD diagrams of Be stars.

\section{PA Variations}

In paper I, we detected variations in the PA of disks during polarimetric outbursts, and speculated that these variations could be indicative of warps, non-equatorial blob injections, or spiral density waves in the inner disk. The left panel of Fig. 2 depicts deviations in the mean PA during polarimetric outbursts of $\pi$ Aqr. As shown in the middle panel, we model a density enhancement on one side (orange) of the disk and a decrement on the opposing side (black) using HDUST. The result of computing the polarization from different viewing angles in a counter-clockwise motion are shown in the right panel of Fig. 2. This model suggests that asymeteric material being ejected from the star can potentially match observed deviations in the PA.

\section{References}

Carciofi, A. C. \& Bjorkman, J. E. 2006, ApJ, 639, 1081

de Wit, W. J., Lamers, H. J. G. L. M., Marquette, J. B., \& Beaulieu, J. P. 2006, A\&SA, 456, 1027

Wisniewski, J. P., Draper, Z. H., Bjorkman, K. S., Meade, M. R. et al. 2010, ApJ, 709, 1306 (paper I) 